PAPER

\title{
Cognitive performance of patients with mesial temporal lobe epilepsy and incidental calcified neurocysticercosis
}

\author{
V C Terra-Bustamante, É R Coimbra, K O Rezek, S R Escorsi-Rosset, R Guarnieri, C L Dalmagro, \\ L M Inuzuka, M M Bianchin, L Wichert-Ana, V Alexandre, O M Takayanagui, D Araújo, \\ A C dos Santos, C G Carlotti, R Walz, H J Markowitsch, A C Sakamoto
}

J Neurol Neurosurg Psychiatry 2005;76:1080-1083. doi: 10.1136/jnnp.2004.048934

See end of article for authors' affiliations

......................

Correspondence to:

Dr V C Terra Bustamante, Department of Neurology, CIREP, Ribeirão Preto School of Medicine, Campus Universitário, Ribeirão Preto, CEP 14049-900, Brazil; vctbusta@rnp.fmrp.usp.br

Received 6 July 2004 In revised form

26 October 2004

Accepted

19 November 2004

\begin{abstract}
Objectives: Although chronic calcified neurocysticercosis (NCC) has been considered a major cause of symptomatic epilepsy in developing countries, it can also be an incidental pathological finding in epileptic patients from endemic regions. The mechanisms of brain plasticity occurring in patients with NCC during and after the inflammatory process related to the parasite infection, death, degeneration, and calcification within the host brain might be an independent factor for cognitive impairment in patients with NCC and epilepsy. In order to assess this possibility cognitive performance of patients with mesial temporal lobe epilepsy related to hippocampal sclerosis (MTLE-HS) with and without NCC was investigated through structured neuropsychological testing.

Methods: Cognitive performance of long term MTLE-HS patients with (HS-NCC group, $n=32$ ) and without NCC (HS only, $n=48$ ) was compared. Imbalances between the two groups with respect to clinical, demographic, neuroimaging, and electrophysiological variables were adjusted by linear multiple regression analysis and Bonferroni correction for multiple tests.

Results and conclusions: There were no cognitive performance differences between HS-NCC and HS only patients, leading to the conclusion that chronic calcified NCC per se does not aggravate the cognitive performance of patients with long term MTLE-HS.
\end{abstract}

$\mathrm{T}$ hree quarters of the estimated 50 million people with epilepsy live in poor countries of the world. ${ }^{12}$ Neurocysticercosis (NCC) is generally considered a major cause of symptomatic epilepsy in developing countries, ${ }^{23}$ including Brazil. ${ }^{45}$ However, owing to its high prevalence in endemic regions, it is possible that chronic NCC could be an incidental finding in a considerable number of epileptic patients from these areas. ${ }^{56}$ Aside from epileptic attacks, other frequently underdiagnosed symptoms of NCC are amnesia, apathy, emotional instability, and hallucinations. ${ }^{1}$ Neuropsychiatric symptoms can vary according to the number of lesions, host inflammatory response, and parasite viability. $^{7-9}$ In an uncontrolled study, Forlenza et al suggested that NCC could also be an important cause of cognitive impairment. In contrast, Boppré et $a l_{,}{ }^{4}$ in a recent case-control study, observed similar cognitive performance in focal epilepsy patients with and without chronic NCC. The limitations of their study were the lack of high resolution MRI studies and of ictal recordings, making the precise diagnosis of the epileptic syndrome and the establishment of the causal relationship between NCC and epilepsy difficult.

Approximately $20 \%$ of the epilepsies are medically intractable, and of these, mesial temporal lobe epilepsy related to hippocampal sclerosis (MTLE-HS) is the most common form of surgically remediable epileptic syndrome. ${ }^{10}$ The hippocampus, which is extensively connected to several neocortical and subcortical structures, is essentially involved in episodic and declarative memory, ${ }^{11}$ and patients with hippocampal sclerosis frequently present with impairment of memory. ${ }^{12}{ }^{13}$ In epileptic patients with NCC, the inflammatory process related to the parasite infection and its death, degeneration, and calcification within the host brain could potentially be an independent and additional factor for cognitive impairment. ${ }^{14}$ In order to address this issue, we investigated whether the presence of chronic NCC is a risk factor for cognitive impairment in patients with MTLE-HS. We decided to investigate patients with MTLE-HS because it is a homogeneous syndrome, well defined by clinical, neurophysiological, neuropathological, and cognitive characteristics.

\section{MATERIALS AND METHODS}

Clinical, demographic, neurophysiological, and neuroimaging data

We studied 80 consecutive patients with MTLE-HS surgically treated under the Ribeirão Preto Epilepsy Surgery Program, between January 1996 and December 1998. All patients had seizures occurring at least monthly despite adequate use of antiepileptic drugs. The study was previously approved by the ethics committee of our institution.

The inclusion criteria have been published previously ${ }^{14} 15$ and included: (a) seizure semiology consistent with MTLE, usually with epigastric, autonomic or psychic auras, followed by behavioural arrest, progressive clouding of consciousness, oroalimentary and manual automatisms, and autonomic phenomena; $(b)$ anterior and mesial temporal lobe interictal spikes; (c) no lesion other than atrophy and increased signal in the hippocampal formation on high resolution MRI, except for calcified NCC lesions (see below, NCC diagnosis); (d) histopathological findings consistent with hippocampal sclerosis (HS); (e) pre-surgical investigation consistent with unilateral temporal lobe seizure onset; (clinical, electrophysiological, neuroimaging, and histopathological results); and $(f)$ at least 1 year of postoperative follow up. The exclusion criteria were: $(a)$ focal neurological abnormalities on physical examination; $(b)$ generalised or extratemporal

Abbreviations: HS, hippocampal sclerosis; MTLE, mesial temporal lobe epilepsy; MTLE-HS, mesial temporal lobe epilepsy related to hippocampal sclerosis; NCC, neurocysticercosis 
interictal EEG spikes; and (c) clinical signs or symptoms of antiepileptic drug toxicity.

Although NCC lesions could be a potential source of seizures and a risk factor for worse outcome after surgery, we had previously observed in the same group of patients ${ }^{6}$ no differences in seizure outcome of MTLE-HS patients with and without NCC, thus reducing the possibility of dual pathology in the present sample.

Patients were evaluated by an experienced multidisciplinary team and their pre-surgical protocol included a detailed clinical history and neurological examination, interictal and ictal video EEG analysis, structural and functional imaging, psychiatric evaluation, neuropsychological testing, and, when appropriate, an intracarotid amobarbital test (Wada's test) for memory and speech representation.

Clinical characteristics included sex, ethnicity, age at surgery, age of epilepsy onset (recurrent seizures), duration of epilepsy, history of initial precipitating insult, antiepileptic drug use and its serum level at the time of neuropsychological evaluation, and monthly complex partial seizure frequency at the time of neuropsychological evaluation. It also included family history of epilepsy, which was defined as positive when at least one of the first or second degree relatives had had at least two spontaneous seizures during their life.

All patients had video EEG recording (Vangard System) performed through closed spaced scalp electrodes plus sphenoidal electrodes. Interictal spikes were assessed by visual analysis of 5 minutes automatically saved EEG samples per hour and classified as: (a) unilateral interictal spikes, if most of the spikes (90\% or more) occurred exclusively in one temporal lobe; or $(b)$ bilateral interictal spikes, if they arose independently from both temporal lobes ( $<90 \%$ in the predominant temporal lobe).

In addition to CT scanning, all patients underwent high resolution MRI ( $1.5 \mathrm{~T}$; Siemens Vision) with special protocols for MTLE-HS. Based on blinded visual MRI analysis by two experienced neuroradiologists (DA and ACS), MTLE-HS was classified as: (a) unilateral, when hippocampal atrophy (on Tl weighted, proton density scans) and/or increased signal (on T2 weighted, or fluid attenuated inversion recovery sequences) were observed only on one side (left or right); or (b) bilateral, when the hippocampal alterations were observed on both sides.

Patients were classified into two groups according to the presence (HS-NCC group, $\mathrm{n}=32$ ) or absence (HS only group, $\mathrm{n}=48$ ) of chronic NCC. Diagnosis of NCC was based on clinical history, known epidemiological data, and CT scans. ${ }^{17}$

The antiepileptic drugs schema at the time of the cognitive assessment included carbamazepine, phenytoin, valproic acid, or phenobarbital, alone or in combination with a benzodiazepine. Serum antiepileptic drugs levels were also determined at that time, and no patient presented signs or symptoms suggestive of intoxication.

\section{Psychometric measurement of cognitive abilities}

Patients underwent a full neuropsychological evaluation, and for the purpose of the present work, we included 19 cognitive tests. Among the IQ subtests from the Wechsler Adult Intelligence Scale-Revised ${ }^{18}$ we analysed the subtests comprehension, similarities, arithmetic, vocabulary, picture completion, and block design. Memory tests included logical memory I and II, and visual reproduction I and II of the Wechsler Memory Scale-Revised. ${ }^{19}$ We also analysed the Rey Auditory Verbal Learning Test (RAVLT), RAVLT retention, and RAVLT delayed recall (30 minutes) ${ }^{20}$; the Rey Visual Design Learning Test (RVDLT), RVDLT retention, and RVDLT delayed recall (30 minutes); and copy of Rey-Osterrieth Complex Figure and its delayed recall (30 minutes). ${ }^{20}$ In addition to the IQ subtests, pre-morbid skills of the patients were also indirectly estimated on the basis of their educational level.

\section{Statistical analysis}

Clinical variables included sex, age at neuropsychological assessment, age of epilepsy onset (recurrent seizures), duration of epilepsy (at the time of neuropsychological assessment), years of education, occupation, positive history for initial precipitating insult, positive family history of seizures in the first and second degree offspring, monthly complex partial seizure frequency in the year before the cognitive evaluation, manual dominance, side of surgery, MRI findings (unilateral or bilateral hippocampal sclerosis), and interictal spikes (unilateral or bilateral). Patients were considered under monotherapy if they were using only one antiepileptic drug. Patients using two or more antiepileptic drugs, including benzodiazepines, were classified as being under polytherapy. They were also classified in those with or without benzodiazepine association. Cognitive performance and other continuous variables were analysed by Student's $t$ test or the Mann-Whitney test, depending on the normality of the distribution of the data. Categorical variables were analysed by $\chi^{2}$ or Fisher's exact tests.

Firstly, we identified possible imbalances among the clinical, demographic, electrophysiological, and neuroimaging variables between patients with and without NCC. Variables in which differences between HS only and HS-NCC disclosed levels of significance $<0.20$ were included in the linear multiple regression.

In the second step, we compared the cognitive performance among all neuropsychological tests between HS-NCC and HS only patients. Neuropsychological variables showing $\mathrm{p}<0.20$ comparing the presence of NCC lesions were also identified. To isolate the variables (clinical, demographic, electrophysiological, and neuroimaging) that significantly contributed to the variance of the cognitive performance in patients with and without NCC lesions, we performed a linear multiple regression test. For these analyses, the cognitive tests were considered the dependent variable. The categorical clinical, demographic, electrophysiological, and neuroimaging variables and the study group were considered fixed factors. The continuous variables, years of education, and serum levels of each antiepileptic drugs used were considered covariates. After the linear multiple regression, association between the cognitive performance and the presence of NCC lesions for $\mathrm{p}<0.01$ was considered significant. This more stringent criterion of significance was based on the Bonferroni adjustment for multiple tests. ${ }^{1721}$ Statistical analysis was performed using the SPSS program (version 10.0; SPSS, Chicago, IL, USA).

\section{RESULTS}

There were 41 male $(51 \%)$ patients; 73 patients $(91 \%)$ were right handed. Family history of epilepsy was positive in 53 $(66 \%)$ patients and $58(72 \%)$ were unskilled or not in employment. Surgery was performed in the left temporal lobe in $53(66 \%)$ patients. Two patients $(2.5 \%)$ disclosed bilateral HS on MRI analysis while interictal spikes were bilateral in $25(31 \%)$ patients. There were 17 patients $(21 \%)$ under polytherapy, and $42(52 \%)$ with benzodiazepine association. The mean (SD) number of NCC lesions in the HS-NCC group was $3(4)$. Fifteen patients $(48.4 \%)$ had a single lesion, 11 (35.5\%) had 2 or 3 lesions, $3(9.6 \%)$ had $4-8$ lesions, and 2 patients $(6.4 \%)$ had 16 and 18 lesions, respectively. The single NCC lesions were distributed in the frontal (three patients), temporal (two), parietal (two) and occipital lobes (six), and in basal ganglia (two). Multiple NCC lesions were distributed in two or more lobes as follows: frontal and parietal (five 
Table 1 Cognitive performance of MTLE-HS patients with (HS-NCC) and without (HS only) NCC

\begin{tabular}{|c|c|c|c|c|c|c|c|}
\hline \multirow[b]{2}{*}{ Cognitive tests } & \multicolumn{2}{|c|}{ All patients } & \multicolumn{2}{|c|}{ Groups HS-NCC } & \multicolumn{2}{|c|}{ HS only } & \multirow[b]{2}{*}{$\mathbf{p}$} \\
\hline & $\mathbf{n}$ & Mean (SD) & $n$ & Mean (SD) & $n$ & Mean (SD) & \\
\hline Comprehension & 79 & $8.1(2.5)$ & 32 & $7.1(2.38)$ & 47 & $8.6(2.3)$ & 0.006 \\
\hline Similarities & 79 & $6.7(1.8)$ & 32 & $6.2(1.6)$ & 47 & $8.7(2.3)$ & 0.11 \\
\hline Vocabulary & 80 & $6.8(1.6)$ & 32 & $6.4(1.7)$ & 48 & $7.1(1.5)$ & 0.06 \\
\hline Blocks & 80 & $7.2(2.3)$ & 32 & $6.4(2.1)$ & 48 & $7.8(2.7)$ & 0.008 \\
\hline Digit span & 79 & $5.3(2.0)$ & 32 & 4.8 & 47 & 5.75 & 0.03 \\
\hline Inverted digit span & 79 & $4.4(1.8)$ & 32 & 3.9 (1.5) & 47 & $4.72(2.0)$ & 0.04 \\
\hline LMI & 80 & $17.8(8.4)$ & 32 & $15.6(6.7)$ & 48 & $19.3(9.2)$ & 0.04 \\
\hline LM II & 80 & $11.8(8.7)$ & 32 & $9.6(7.5)$ & 48 & $13.3(9.2)$ & 0.05 \\
\hline VR I & 78 & $31.3(6.9)$ & 30 & $29.8(6.9)$ & 48 & $32.2(6.8)$ & 0.13 \\
\hline VR II & 78 & $20.4(11.7)$ & 30 & $17.7(10.2)$ & 48 & $22.0(12.3)$ & 0.11 \\
\hline Copy of ROCF & 76 & $23.7(45.5)$ & 30 & $17.8(41.4)$ & 46 & $27.5(48.0)$ & 0.37 \\
\hline RAVLT & 80 & $49.3(11.2)$ & 32 & $47.7(12.2)$ & 48 & $50.4(10.6)$ & 0.30 \\
\hline RAVLT retention & 80 & $9.2(3.6)$ & 32 & $9.1(3.9)$ & 48 & $9.2(3.4)$ & 0.89 \\
\hline RAVLT delayed recall & 79 & $8.8(4.3)$ & 32 & $8.9(4.3)$ & 48 & $8.7(4.2)$ & 0.87 \\
\hline RVDLT & 79 & $27.8(13.1)$ & 32 & $25.0(12.5)$ & 47 & 29.7 (13.2) & 0.12 \\
\hline RVDLT retention & 79 & $6.8(3.7)$ & 32 & $6.2(3.5)$ & 47 & $7.1(3.7)$ & 0.28 \\
\hline RVDLT delayed recall & 80 & $6.5(3.6)$ & 32 & $6.1(3.4)$ & 48 & $6.8(3.7)$ & 0.33 \\
\hline ROCF delayed recall & 78 & $53.7(66.9)$ & 32 & $60.2(81.5)$ & 48 & $49.3(55.7)$ & 0.5 \\
\hline
\end{tabular}

LM I, Logical Memory I; LM II, Logical Memory II; VR I, Visual Reproduction I; VR II, Visual Reproduction II; RAVLT, Rey Auditory Verbal Learning Test; RVDLT, Rey Visual Design Learning Test; ROCF, Rey-Osterrieth Complex Figure.

patients), frontal and occipital (two), parietal and occipital (four), frontal, temporal, and occipital (one), frontal, parietal, and occipital (one), frontal and occipital lobes, and basal ganglia (one), frontal, parietal, and occipital lobes, and basal ganglia (one), and frontal, temporal, parietal, and occipital lobes, and basal ganglia (one). There were no statistically significant differences among these clinical, demographic, radiological, and neurophysiological variables between the HS-NCC and HS only groups. The analysis of the cognitive performance of the two groups of patients is given in table I. Patients with HS-NCC disclosed a significantly lower performance in the comprehension $(p=0.006)$, blocks $(p=0.008)$, digit span $(p=0.03)$, inverted digit span $(p=0.04)$, LM I $(p=0.04)$, and LM II $(p=0.05)$ tests. There were no statistical differences in test performances when comparing patients with single and multiple NCC lesions $(\mathrm{p}>15)$.

\section{DISCUSSION}

In the present study, we analysed patients with calcified NCC and MTLE-HS. We assumed that the aetiology of punctuate calcified lesions was NCC, based on the high prevalence of NCC in our region, ${ }^{56}$ and on several previous reports that have established criteria for differentiating cysticerci from other lesions, such as tuberculosis and fungal infections. ${ }^{1461417}$ It is well known that these infections do not resolve into calcified lesions unless specific therapy is applied. ${ }^{6}$ None of our patients had been treated with any anti-tuberculous or antifungal drugs, or had any circumstantial evidence of these infections elsewhere in their body.

Cognitive performance of patients with MTLE-HS with or without calcified NCC was similar for the majority of tests analysed, for both single and multiple lesions. These findings are in agreement with those reported by Boppré et al, who showed that calcified NCC did not correlate with neuropsychological performance in patients with focal epilepsy. ${ }^{4}$ Although some subtests (comprehension, blocks, digit span, inverted digit span, logical memory) had initially shown significant differences between MTLE-HS patients with and without NCC lesions, only the educational level remained significant after adjusting for clinical, demographic, neuroimaging, and electrophysiological imbalances in a multilinear regression analysis, thus suggesting the possibility that the low performance of patients with incidental NCC could be due to their lower education level, and not the NCC infection itself $(p<0.001)$. The lower education of patients with NCC lesions can be directly related to their worse socioeconomic levels and consequently higher risk of parasitic infection.

The potential harmful effect of several other variables on the brain functions of patients with epilepsy, including ictal and interictal discharges, neuropathology, antiepileptic drugs, and psychosocial factors, has been extensively discussed in the clinical literature. ${ }^{22}$ All these variables can interfere with the neuronal and glial receptor and postreceptor mechanisms of integrated cortical and subcortical brain regions, and hormonal and neurohumoral modulatory systems involved in cognitive procedures and behavioural responses. ${ }^{23-25}$

The present study clearly indicates that inflammatory processes related to parasite infection, death, degeneration, and calcification in the host brain do not significantly aggravate the cognitive deficits observed in medically refractory MTLE-HS patients.

\section{ACKNOWLEDGEMENTS}

This work was supported by the Volkswagen Foundation (Partnerschaftsprogramm: Forschungsvorhaben mit natur-, ingenieur- und wirtschaftswissenschaftlichen Instituten in Afrika, Asien und Lateinamerika) (R Walz, M M Bianchin and H J Markowitsch). H J Markowitsch was a visiting professor at the Centro de Cirurgia de Epilepsia do Estado de Santa Catarina, Hospital Governador Celso Ramos, Florianópolis, SC. M M Bianchin is supported by FAPESP. O M Takayanagui is supported by CNPq (no. 300937/2003-2)

\section{Authors' affiliations \\ V C Terra-Bustamante, É R Coimbra, K O Rezek, S R Escorsi-Rosset, R Guarnieri, C L Dalmagro, L M Inuzuka, M M Bianchin, L Wichert-Ana, V Alexandre, O M Takayanagui, A C Sakamoto, Epilepsy Surgery Center (CIREP), Department of Neurology, Ribeirão Preto School of Medicine, University of São Paulo, Brazil \\ D Araújo, A C dos Santos, Department of Radiology, Ribeirão Preto School of Medicine, University of São Paulo, Brazil \\ C G Carlotti, Neurosurgery Section, Department of Surgery and \\ Anatomy, Ribeirão Preto School of Medicine, University of São Paulo, Brazil \\ R Walz, Epilepsy Surgery Center, Hospital Governador Celso Ramos, Florianópolis, Santa Catarina, Brazil}


R Walz, Laboratory of Experimental Surgery, University of Vale do Itajaí Medical School, Brazil; andlnternal Medicine Department, Medicine School of Universidade Federal de Santa Catarina, Florianópolis, Santa Catarina, Brazil

H J Markowitsch, Arbeitseinheit Physiologische Psychologie, Universität Bielefeld, Germany

Competing interests: none declared

\section{REFERENCES}

1 Pal DK, Carpio A, Sander JW. Neurocysticercosis and epilepsy in developing countries. J Neurol Neurosurg Psychiatry 2000;68:137-43.

2 Sander JW, Shorvon SD. Epidemiology of the epilepsies. J Neurol Neurosurg Psychiatry 1996;61:433-43.

3 Carpio A, Escobar A, Hauser WA. Cysticercosis and epilepsy: a critical review. Epilepsia 1998;39:1025-40.

4 Boppre MC, Wille PR, Guarnieri R, et al. Cognitive performance of patients with epilepsy and calcified neurocysticercotic lesions: A case-control study. Epilepsy Behav 2001;2:558-62.

5 Takayanagui OM. Therapy for neurocysticercosis. Expert Rev Neurotherapeutics 2004;4:129-39.

6 Leite JP, Terra-Bustamante VC, Fernandes RM, et al. Calcified neurocysticercotic lesions and postsurgery seizure control in temporal lobe epilepsy. Neurology 2000;55:1485-91.

7 Forlenza OV, Filho AH, Nobrega JP, et al. Psychiatric manifestations of neurocysticercosis: a study of 38 patients from a neurology clinic in Brazil. I Neurol Neurosurg Psychiatry 1997;62:612-16.

8 McCormick GF, Zee CS, Heiden J. Cysticercosis cerebri. Review of 127 cases. Arch Neurol 1982;39:534-9.

9 Garcia HH, Gonzalez AE, Evans CA, et al. Taenia solium cysticercosis. Lancet 2003:361:547-56.

10 Engel J Jr. Surgery for seizures. N Engl J Med 1996;334:647-52.

11 Tulving E, Markowitsch HJ. Episodic and declarative memory: role of the hippocampus. Hippocampus 1998;8:198-204.
12 Hermann BP, Seidenberg M, Schoenfeld J, et al. Empirical techniques for determining the reliability, magnitude, and pattern of neuropsychological change after epilepsy surgery. Epilepsia 1996;37:942-50.

13 Hermann BP, Seidenberg M, Schoenfeld J, et al. Neuropsychological characteristics of the syndrome of mesial temporal lobe epilepsy. Arch Neurol 1997; 54:369-76.

14 Nash TE, Del Brutto OH, Butman JA, et al. Calcific neurocysticercosis and epileptogenesis. Neurology 2004;62:1934-8.

15 Velasco TR, Wichert-Ana L, Leite JP, et al. Accuracy of ictal SPECT in mesial temporal lobe epilepsy with bilateral interictal spikes. Neurology 2002;59:266-71.

16 Walz R, Castro RM, Velasco TR, et al. Surgical outcome in mesial temporal sclerosis correlates with prion protein gene variant. Neurology 2003;61:1204-10.

17 Del Brutto OH, Rajshekhar V, White AC Jr, et al. Proposed diagnostic criteria for neurocysticercosis. Neurology 2001;57:177-83.

18 Wechsler D. Wechsler adult intelligence scale-revised. New York: The Psychological Corporation, 1981.

19 Wechsler D. Wechsler memory scale-revised. New York: The Psychological Corporation, 1987.

20 Spreen O, Strauss E. A compendium of neuropsychological tests. New York: Oxford University Press, 1998.

21 Perneger TV. What's wrong with Bonferroni adjustments? BMJ 1998;316:1236-8.

22 Kwan P, Brodie M. Neuropsychological effects of epilepsy and antiepileptic drugs. Lancet 2001;357:216-22.

23 Izquierdo I, Medina JH, Bianchin M, et al. Memory processing by the limbic system: role of specific neurotransmitter systems. Behav Brain Res 1993;58:91-8.

24 Milner B, Squire LR, Kandel ER. Cognitive neuroscience and the study of memory. Neuron 1998;20:445-68.

25 Walz R, Roesler R, Quevedo J, et al. Time-dependent impairment of inhibitory avoidance retention in rats by postraining infusion of a mitogen-activated protein kinase inhibitor into cortical and limbic structures. Neurobiol Learn Mem 2000;73:11-20 